\title{
Influence of Random Excitations on Acoustic Instabilities in Combustion Chambers
}

\author{
V. S. Burnley* \\ U.S. Air Force Research Laboratory, Edwards Air Force Base, California 93524 \\ and \\ F. E. C. Culick ${ }^{\dagger}$ \\ California Institute of Technology, Pasadena, California 91125
}

\begin{abstract}
Although flows in combustors contain considerable noise, arising from several kinds of sources, there is a sound basis for treating organized oscillations as distinct motions. That has been an essential assumption incorporated in virtually all treatments of combustion instabilities. However, certain characteristics of the organized or deterministic motions seem to have the nature of stochastic processes. For example, the amplitudes in limit cycles always exhibit a random character, and even the occurrence of instabilities seems occasionally to possess some statistical features. Analysis of nonlinear coherent motions in the presence of stochastic sources is, therefore, an important part of the theory. We report a few results for organized oscillations in the presence of noise. The most significant deficiency is that, because of the low level of current understanding, the stochastic sources of noise are modeled in ad hoc fashion and are not founded on a solid physical basis appropriate to combustion chambers.
\end{abstract}

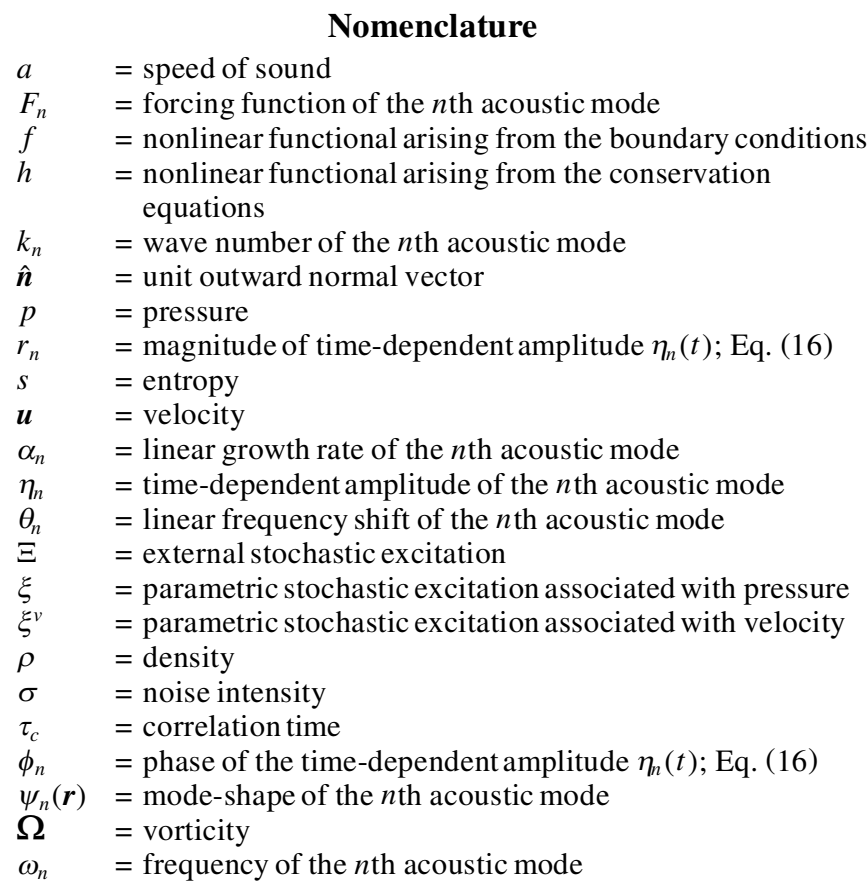

\section{Subscripts}

$\begin{array}{ll}a & =\text { acoustic waves } \\ s & =\text { entropy waves } \\ \Omega & =\text { vorticity waves }\end{array}$

Superscripts
$\mathrm{NL} \quad=$ nonlinear
$\left(^{-}\right) \quad=$ mean quantity
$\left(^{\prime}\right) \quad=$ fluctuating quantity
$(\cdot) \quad=$ time derivative

\footnotetext{
Received 23 December 1997; revision received 21 October 1999; accepted for publication 22 November 1999. This material is declared a work of the U.S. Government and is not subject to copyright protection in the United States.

${ }^{*}$ Research Engineer, Propulsion Directorate. Senior Member AIAA.

${ }^{\dagger}$ Richard L. and Dorothy M. Hayman Professor of Mechanical Engineering and Professor of Jet Propulsion. Fellow AIAA.
}

\section{Introduction}

C OMBUSTION chambers are inherently noisy environments. This is apparent from inspection of the power spectra of pressure records from test firings, as well as from simply listening to the tests. When an organized oscillation, that is, a combustion instability, is present, the power spectrum exhibits well-defined peaks in addition to background noise over the entire range of frequencies. Substantial noise sources in rocket motors include flow separation, turbulence, and combustion processes. It is expected that the presence of noise will affect in some way the amplitudes and possibly the qualitative behavior of coherent oscillations. Analysis of coherent oscillations in the presence of noise is, therefore, an important part of the theory. Nonetheless, there is a sound basis for treating instabilities as distinct motions, and much progress has been made using this assumption.

Coherent oscillations in combustion chambers can generally be grouped into two categories based on the linear stability of the system. The two general types are spontaneous oscillations and pulsed oscillations. A spontaneous oscillation occurs in a linearly unstable system such that any perturbation of the system grows exponentially in time. Under the influence of nonlinear effects, the pressure field may reach a periodic motion, or limit cycle. This type of instability is also known as an intrinsic instability or a soft excitation. In the field of dynamical systems, the change of behavior from a linearly stable steady state to a stable path of periodic solutions is called a supercritical bifurcation.

A pulsed oscillation, on the other hand, is a true nonlinear instability of a linearly stable system. Small perturbations in the pressure field decay exponentially to zero, whereas larger perturbations may lead to stable or unstable periodic motions. Common terminology used to describe this type of oscillationincludestriggered instability, hard excitation, and subcritical bifurcation. Previous works, for example, Yang et al., ${ }^{1}$ Paparizos and Culick, ${ }^{2}$ and Jahnke and Culick, ${ }^{3}$ have convincinglyshown that nonlineargasdynamics alone does not contain the possibility of pulsed oscillations. Part of the purpose of the present investigation is to determine if noise processes could be responsible for the occurrence of pulsed oscillations.

Although the effects of noise or random excitations have been studied extensively in other fields (such as signal processing, economics and finance, and physics, to name a few ${ }^{4}$ ), only a small amount of work has been done on the interactions between noise and acoustic instabilities. Strahle, ${ }^{5,6}$ Muthukrishnan et al., ${ }^{7}$ and Hedge and Strahle ${ }^{8}$ studied combustion noise and possible interaction between noise and instabilitiesover a number of years. They concluded that the coupling between combustion noise and acoustic waves is 
not strong enough to drive large-amplitudeoscillations.$^{5-8}$ Deur and Hessler ${ }^{9}$ investigated external excitations as a possible explanation for large-amplitudeoscillationsin combustion chambers. As we will show, inclusion of noise will lead to external excitations that could theoretically produce large-amplitude forced oscillations as Deur and Hessler have suggested. However, there is a fundamental difference in the transient behavior of forced oscillations and self-excited oscillations. Experimental data from rocket motors almost always display the common exponential growth that is characteristic of a self-excited oscillation. Therefore, we believe that large-amplitude oscillationscommonly found in combustion chambers are not forced and that the external excitation provided by combustionnoise is quite small, leading only to very low-amplitude forced oscillations.

Culick et al. ${ }^{10}$ studied the influence of noise on combustion instabilities, but only for a very simple case: two acoustic modes with noise present only in the first mode. In addition, the formulation was flawed, and the form of the resulting noise terms is not quite correct. Clavin et al. ${ }^{11}$ studied the influence of turbulence on instabilities in liquid rocket motors. Using only one mode in the analysis and thirdorder nonlinearities, it was reported that the inclusion of noise can lead to the possibility of pulsed oscillations. It is a well-known result that a single third-order equation may produce a subcritical bifurcation. When more acoustic modes are considered, this may not be the case, as demonstrated by Yang et al. ${ }^{1}$ for third-order gasdynamics. Therefore, the results of Clavin et al. ${ }^{11}$ may not be applicable in general.

The present analysis is an extension of the previous work by Culick et al. ${ }^{10}$ The approximate analysis used here has been covered in other works, and so only a brief overview of the method is presented; for more details, see, for example, the review by Culick. ${ }^{12}$ This analysis will be extended by decomposing the unsteady flowfield into acoustic and nonacoustic parts based on the approach followed by Chu and Kovásznay. ${ }^{13}$ That tactic produces a formal representation of the noise terms in the governing equations. Finally, we will simplify the equations to study more conveniently the possible influences of noise on acoustic instabilities.

\section{Development of the Approximate Analysis}

To keep the analysis as general as possible, the formulation begins with the conservationequations for two-phase flow. These equations are rewritten in an equivalent form for a single medium having the mass-averaged properties of the two phases. Subsequently, a wave equation for the pressure is developed, along with the corresponding boundary condition:

$$
\begin{gathered}
\nabla^{2} p^{\prime}-\frac{1}{\bar{a}^{2}} \frac{\partial^{2} p^{\prime}}{\partial t^{2}}=h \\
\hat{\boldsymbol{n}} \cdot \nabla p^{\prime}=-f
\end{gathered}
$$

The functions $h$ and $f$ are linear and nonlinear functions of the pressure and velocity perturbations. As an approximation, these perturbations are expanded as a synthesis of classical acoustic modes with time-varying amplitudes:

$$
\begin{aligned}
& p^{\prime}(\boldsymbol{r}, t)=\bar{p} \sum_{n=1}^{\infty} \eta_{n}(t) \psi_{n}(\boldsymbol{r}) \\
& \boldsymbol{u}^{\prime}(\boldsymbol{r}, t)=\sum_{n=1}^{\infty} \frac{\dot{\eta}_{n}(t)}{\bar{\gamma} k_{n}^{2}} \nabla \psi_{n}(\boldsymbol{r})
\end{aligned}
$$

where $\eta_{n}(t)$ is the time-dependent amplitude of the $n$th classical acoustic mode. After substituting Eq. (3) in Eq. (1), the equations are spatially averaged, resulting in a system of ordinary differential equations describing the amplitudes of the acoustic modes:

$$
\frac{\mathrm{d}^{2} \eta_{n}}{\mathrm{~d} t^{2}}+\omega_{n}^{2} \eta_{n}=F_{n}
$$

where $\omega_{n}=\bar{a} k_{n}$ and

$$
F_{n}=-\frac{\bar{a}^{2}}{\bar{p} E_{n}^{2}}\left\{\int \psi_{n} h \mathrm{~d} V+\oint \psi_{n} f \mathrm{~d} S\right\}
$$

The problem has been reduced to solving Eq. (5) for the amplitudes $\eta_{n}(t)$. This approach is very general and can accommodate all damping and amplification mechanisms. The most difficult part of the problem is in the identification and modeling of the important physical processes.

\section{Splitting the Unsteady Flowfield into Acoustic, Vortical, and Entropic Modes of Propagation}

Fluctuations in a compressible fluid can be decomposed into three types of waves: acoustic waves, vorticity waves, and entropy waves. A thorough discussion of this idea is presented by Chu and Kovásznay. ${ }^{13}$ In the limit of small amplitudes, the three types of waves propagate independently in a uniform mean flow, but are coupled when the mean flow is nonuniform. ${ }^{14}$ For example, the pressure in an acoustic wave is changed slightly by the presence of a vorticity or entropy wave if the mean flow is not uniform. Coupling between the types of waves may also occur at the boundaries of the chamber.

Although noise is detected as pressure waves, the sources of noise are associated with the presence of vorticity fluctuations, for example, turbulence, flow separation, etc., and entropy or nonisentropic temperature fluctuations. Therefore, decomposing the unsteady flowfield into the three types of waves allows both noise and acoustic instabilities to be handled in the same analytical framework discussed in Sec. I. The contributions from vorticity and entropy waves appear as additional force terms on the right-hand side of the acoustic equation.

Following the analysis of Chu and Kovásznay, ${ }^{13}$ the thermodynamic and kinematic variables can be written as sums of fluctuations in the three waves as follows: $p^{\prime}=p_{a}^{\prime}+p_{\Omega}^{\prime}+p_{s}^{\prime}$, $\boldsymbol{\Omega}=\boldsymbol{\Omega}_{a}^{\prime}+\boldsymbol{\Omega}_{\Omega}+\boldsymbol{\Omega}_{s}, s^{\prime}=s_{a}^{\prime}+s_{\Omega}^{\prime}+s_{s}^{\prime}$, and $\boldsymbol{u}^{\prime}=\boldsymbol{u}_{a}^{\prime}+\boldsymbol{u}_{\Omega}^{\prime}+\boldsymbol{u}_{s}^{\prime}$. In general, all of the fluctuations will be nonzero, but not all terms are of the same order. If we restrict the analysis to small-amplitude motions and ignore coupling of waves at the boundaries, the three waves have the following characteristics ${ }^{13}$ : acoustic waves, with pressure and velocity fluctuations, no entropy change; vorticity waves, with velocity fluctuations, no pressure or entropy changes; and entropy waves, entropy and velocity fluctuations, no pressure change (see the Nomenclature). Thus, to zeroth order, the fluctuations in the three waves are given by

$$
\begin{gathered}
p^{\prime}=p_{a}^{\prime} \\
\boldsymbol{\Omega}=\Omega_{\Omega}^{\prime} \\
s^{\prime}=s_{s}^{\prime} \\
\boldsymbol{u}^{\prime}=\boldsymbol{u}_{a}^{\prime}+\boldsymbol{u}_{\Omega}^{\prime}+\boldsymbol{u}_{s}^{\prime}
\end{gathered}
$$

An equation for the density fluctuation is obtained by expanding the formula for the entropy of a perfect gas:

$$
\rho^{\prime} / \bar{\rho}=(1 / \bar{\gamma})\left(p_{a}^{\prime} / \bar{p}\right)-\left(1 / \bar{c}_{p}\right) s^{\prime}
$$

As an approximation to the acoustic pressure and velocity perturbations, we will once again use a superposition of the classical acoustic modes so that

$$
p_{a}^{\prime}=\bar{p} \sum_{n=1}^{\infty} \eta_{n}(t) \psi_{n}(\boldsymbol{r}), \quad \boldsymbol{u}_{a}^{\prime}=\sum_{n=1}^{\infty} \frac{\dot{\eta}_{n}(t)}{\bar{\gamma} k_{n}^{2}} \nabla \psi_{n}(\boldsymbol{r})
$$

Substitution of Eq. (7) in the left-hand side of the nonlinear wave Eq. (1), followed by application of Galerkin's method, leads to a set of coupled nonlinear oscillator equations:

$$
\ddot{\eta}_{n}+\omega_{n}^{2} \eta_{n}=F_{n}
$$

where $^{14}$

$$
\begin{aligned}
& -\frac{\bar{p} E_{n}^{2}}{\bar{a}^{2}} F_{n}=\bar{\rho} I_{1}+\frac{1}{\bar{a}^{2}} I_{2}+\bar{\rho} I_{3}+\frac{1}{\bar{a}^{2}} I_{4} \\
& +\int \bar{\rho} \frac{\partial \boldsymbol{u}^{\prime}}{\partial t} \cdot \hat{\boldsymbol{n}} \mathrm{d} S-\int\left(\frac{1}{\bar{a}^{2}} \frac{\partial \mathcal{P}^{\prime}}{\partial t} \psi_{n}+\mathcal{F}^{\prime} \cdot \nabla \psi_{n}\right) \mathrm{d} V
\end{aligned}
$$


$I_{1}=\int\left(\overline{\boldsymbol{u}} \cdot \nabla \boldsymbol{u}^{\prime}+\boldsymbol{u}^{\prime} \cdot \nabla \overline{\boldsymbol{u}}\right) \cdot \nabla \psi_{n} \mathrm{~d} V$

$I_{2}=\frac{\partial}{\partial t} \int\left(\bar{\gamma} p^{\prime} \nabla \cdot \overline{\boldsymbol{u}}+\overline{\boldsymbol{u}} \nabla \cdot p^{\prime}\right) \psi_{n} \mathrm{~d} V$

$I_{3}=\int\left(\boldsymbol{u}^{\prime} \cdot \nabla \boldsymbol{u}^{\prime}+\frac{\rho^{\prime}}{\bar{\rho}} \frac{\partial \boldsymbol{u}^{\prime}}{\partial t}\right) \cdot \nabla \psi_{n} \mathrm{~d} V$

$I_{4}=\frac{\partial}{\partial t} \int\left(\bar{\gamma} p^{\prime} \nabla \cdot \boldsymbol{u}^{\prime}+\boldsymbol{u}^{\prime} \cdot \nabla p^{\prime}\right) \psi_{n} \mathrm{~d} V$

In the original development of the approximate analysis, the zerothorder approximations for the pressure and velocity were used to evaluate $F_{n}$. The same idea will be applied here, although additional contributions to the velocity fluctuation from coupling to vorticity and entropy waves will be included. Once these quantities are substituted in the right-hand side, the set of forced oscillator equations eventually takes the general form (see work by Burnley ${ }^{15}$ for details):

$$
\begin{aligned}
\ddot{\eta}_{n}+\omega_{n}^{2} \eta_{n}=2 \alpha_{n} \dot{\eta}_{n}+2 \omega_{n} \theta_{n} \eta_{n}-\sum_{i=1}^{\infty} \sum_{j=1}^{\infty}\left[A_{n i j} \dot{\eta}_{i} \dot{\eta}_{j}+B_{n i j} \eta_{i} \eta_{j}\right] \\
+\left(F_{n}\right)_{\text {other }}^{\mathrm{NL}}+\sum_{i=1}^{\infty}\left[\xi_{n i}^{v}(t) \dot{\eta}_{i}+\xi_{n i}(t) \eta_{i}\right]+\Xi_{n}(t)
\end{aligned}
$$

Note that the terms $\xi_{n i}^{v}(t) \dot{\eta}_{i}, \xi_{n i}(t) \eta_{i}$, and $\Xi_{n}(t)$ arise directly from $I_{1}, I_{3}$, and $I_{4}$ with the velocities written as fluctuations from vorticity, entropy, and acoustic waves. For example, the formula for the external excitation $\Xi_{n}(t)$ is

$\Xi_{n}(t)$

$$
\begin{aligned}
& =-\frac{\bar{a}^{2} \bar{\rho}}{\bar{p} E_{n}^{2}}\left\{\int\left[\nabla\left(\overline{\boldsymbol{u}} \cdot \boldsymbol{u}_{\Omega}^{\prime}\right)-\boldsymbol{u}_{\Omega}^{\prime} \times \nabla \times \overline{\boldsymbol{u}}-\overline{\boldsymbol{u}} \times \mathbf{\Omega}\right] \cdot \nabla \psi_{n} \mathrm{~d} V\right. \\
& +\int\left[\nabla\left(\overline{\boldsymbol{u}} \cdot \boldsymbol{u}_{s}^{\prime}\right)-\boldsymbol{u}_{s}^{\prime} \times \nabla \times \overline{\boldsymbol{u}}-\overline{\boldsymbol{u}} \times \nabla \times \boldsymbol{u}_{s}^{\prime}\right] \cdot \nabla \psi_{n} \mathrm{~d} V \\
& +\int\left[\boldsymbol{u}_{\Omega}^{\prime} \cdot \nabla \boldsymbol{u}_{\Omega}^{\prime}\right] \cdot \nabla \psi_{n} \mathrm{~d} V+\int\left[\boldsymbol{u}_{\Omega}^{\prime} \cdot \nabla \boldsymbol{u}_{s}^{\prime}\right] \cdot \nabla \psi_{n} \mathrm{~d} V \\
& +\int\left[\boldsymbol{u}_{s}^{\prime} \cdot \nabla \boldsymbol{u}_{\Omega}^{\prime}\right] \cdot \nabla \psi_{n} \mathrm{~d} V \\
& \left.+\int\left(\boldsymbol{u}_{s}^{\prime} \cdot \nabla \boldsymbol{u}_{s}^{\prime}+\frac{\rho_{s}^{\prime}}{\bar{\rho}} \frac{\partial \boldsymbol{u}_{s}^{\prime}}{\partial t}\right) \cdot \nabla \psi_{n} \mathrm{~d} V\right\}
\end{aligned}
$$

This is purely a formal procedure; the terms cannot be calculated at this point but must instead be modeled.

This system of equations is very complex, with many free parameters. For instance, if we truncate the system to $N$ modes, there are $2 N$ linear parameters and an additional $2 N^{2}+N$ unknown functions. To simplify the equations somewhat, we will, therefore, neglect cross-coupling terms in $\xi_{n i}^{v}$ and $\xi_{n i}$, that is, terms with $n \neq i$. These terms may turn out to be important, but neglecting them will allow for easier initial computation of results and will suffice for the purposes here. The simplified set of equations is

$$
\begin{aligned}
\ddot{\eta}_{n}+ & \omega_{n}^{2} \eta_{n}=2 \alpha_{n} \dot{\eta}_{n}+2 \omega_{n} \theta_{n} \eta_{n}-\sum_{i=1}^{\infty} \sum_{j=1}^{\infty}\left[A_{n i j} \dot{\eta}_{i} \dot{\eta}_{j}+B_{n i j} \eta_{i} \eta_{j}\right] \\
& +\left(F_{n}\right)_{\mathrm{other}}^{\mathrm{NL}}+\xi_{n}^{v}(t) \dot{\eta}_{n}+\xi_{n}(t)_{\eta_{n}}+\Xi_{n}(t)
\end{aligned}
$$

\section{Modeling of the Stochastic Sources}

The nondeterministic problem has now been reduced to solving Eq. (15) for the time-dependent amplitudes $\eta_{n}(t)$ that are subjected
Table 1 Linear growth rates and frequency shifts

\begin{tabular}{lcrrr}
\hline \hline & \multicolumn{4}{c}{$n$} \\
\cline { 2 - 5 } Parameter & 1 & \multicolumn{1}{c}{2} & \multicolumn{1}{c}{3} & \multicolumn{1}{c}{4} \\
\hline$\alpha_{n}, \mathrm{~s}^{-1}$ & Free & -324.8 & -583.6 & -889.4 \\
$\theta_{n}, \mathrm{rad} / \mathrm{s}$ & 12.9 & 46.8 & -29.3 & -131.0 \\
\hline \hline
\end{tabular}
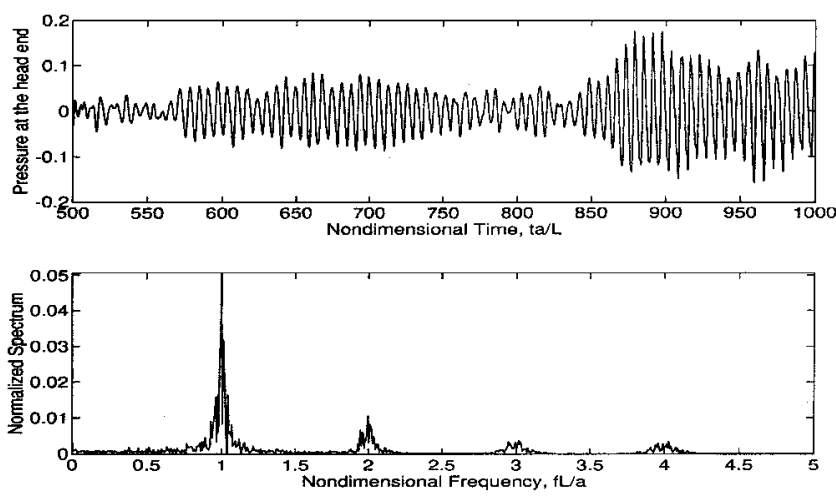

Fig. 1 Sample pressure trace and spectrum for a simulation.

to additive and multiplicative noise. The source terms $\xi_{n}^{v}(t), \xi_{n}(t)$, and $\Xi_{n}(t)$ represent stochastic processes of some sort and are responsible, in this formulation, for the background noise found in the power spectra of test firings. The problem of modeling these processes, however, remains. This requires specification of both the spatial and temporal distribution of the velocity and the entropy. At the present time, no models exist for these fluctuations.

There are several paths that can be followed at this point, which include obtaining approximate representations for the velocity and entropy fluctuations based on experimental data or numerical simulations. The approach that will be taken here is to assume forms for the source terms that are based on observations of experiments. From inspection of the power spectra of test firings, it is apparent that the stochastic processes in real systems are broadband with very small correlation times $\tau_{c}$. (Note that the correlation time is the time above which the autocorrelationfunction is zero. This is a measure of the dependence of the process on its past.) The limit $\tau_{c} \rightarrow 0$ represents a delta correlated process, that is, a process that is totally uncorrelated with itself. It is, thus, interesting to study this limiting case and assume that the stochastic terms are represented by white noise.

The definition of a white noise process is a process whose spectral density is flat, that is, all frequencies are present at the same amplitude. Although such a process cannot occur in a real system, white noise can be a very useful tool for studying real processes that have very small correlation times compared to the macroscopic times of the system. This is true of the random processes and systems of interest. ${ }^{10}$ Therefore, we will approximate $\xi_{n}^{v}, \xi_{n}$, and $\Xi_{n}$ by mutually independent white noise processes with zero mean values. The intensities of these processes will be denoted by $\sigma_{n}^{\xi^{\nu}}, \sigma_{n}^{\xi}$, and $\sigma_{n}^{\Xi}$, respectively.

An example of a simulation with white noise excitations is presented in Fig. 1. A sample trace of the pressure at the head end of the combustion chamber is shown along with the corresponding normalized spectrum. Inspection of the spectrum shows peaks at distinct frequencies that are associated with an acoustic instability, along with broadband background noise. This is characteristic of actual test data of a case when an instability is present. For the simulation shown in Fig. 1, as well as all other results presented here, we restrict the analysis to longitudinal modes in a cylindrical combustion chamber. The values given in Table 1 are used for the linear parameters of the system, and the fundamental frequency $\omega_{1}$ is $5654.87 \mathrm{rad} / \mathrm{s}$.

\section{Results}

Because we are interested in nondeterministic systems, it is natural to use the probability density functions of the amplitudes of acoustic modes to investigate the dynamics of the system. In 
particular, we will use the amplitude $r_{n}$ that is introduced in the following equation:

$$
\eta_{n}(t)=r_{n}(t) \cos \left[\omega_{n} t+\phi_{n}(t)\right]
$$

The amplitude $r_{n}$ is defined as $\sqrt{ }\left(\eta_{n}^{2}+\dot{\eta}_{n}^{2} / \omega_{n}^{2}\right)$ and is a measure of the magnitude of the oscillation. Using $r_{n}$ instead of $\eta_{n}$, quantitative changes in the solution will be more apparent because the expected (or mean) value of $r_{n}$ is nonzero for any nontrivial oscillation, even if the expected value of $\eta_{n}$ is equal to zero. The change of variables from $\left(\eta_{n}, \dot{\eta}_{n}\right)$ to $\left(r_{n}, \phi_{n}\right)$ causes a shift in the expected value of the amplitude for any nontrivial oscillation. This is obvious from the definition of $r_{n}$.

A Monte Carlo method will be used to obtain an approximation to the probability density functions of the acoustic amplitudes. In this method, a series of numerical experiments is conducted, usually in the same manner that one would conduct actual experiments. After the flowfield has become well developed (for example, after 1000 periods of the fundamental mode or so), the amplitudes of the acoustic modes are sampled. The results are then used to construct histograms that, after normalization, approximate the instantaneous probability density functions of the modal amplitudes. The approximation becomes better as the number of experiments is increased.

In the current study, each Monte Carlo simulation will consist of 10,000 numerical experiments. The linear parameters will be fixed throughout a series of experiments, while the initial conditions for the simulations will be varied systematically. In particular, a square initial pulse that is nonzero from $0 \leq x / L \leq 0.25$ will be used. The size of the pressure pulse $p^{\prime} / \bar{p}$ will be varied from 0 to 0.2 linearly so as to include most likely values.

For the initial results, we will use the simplest possible set of equations. The system will be truncated to two modes with noise included explicitly only in the fundamental mode. We will also assume initially that the only nonlinear contributions are associated with gasdynamics. These simplifications were used by Culick et al. ${ }^{10}$ and will allow the effects of each type of stochastic excitation to be determined. We will relax these simplifications later in this section by also including nonlinear contributions from combustion and noise explicitly in more than one mode.

\section{A. Effects of an External Excitation}

The term $\Xi_{1}$ is an external excitation, that is, it does not depend on the current state of the system. As a result, this type of excitation does not change the qualitative behavior of the system from that of the original deterministic system, ${ }^{16}$ that is, no matter how large the intensity, the addition of an external excitation does not change the locations or types of the stable states, or attractors, from those of the original deterministic system. Instead, an external excitation provides only a random perturbationfrom these states; the dynamics of the system are constantly acting to bring a stable system back to a state of equilibrium. The bifurcation diagram for the deterministic system is shown in Fig. 2. Figure 2 demonstrates how the amplitude of the fundamental acoustic mode changes as the linear growth rate is varied.

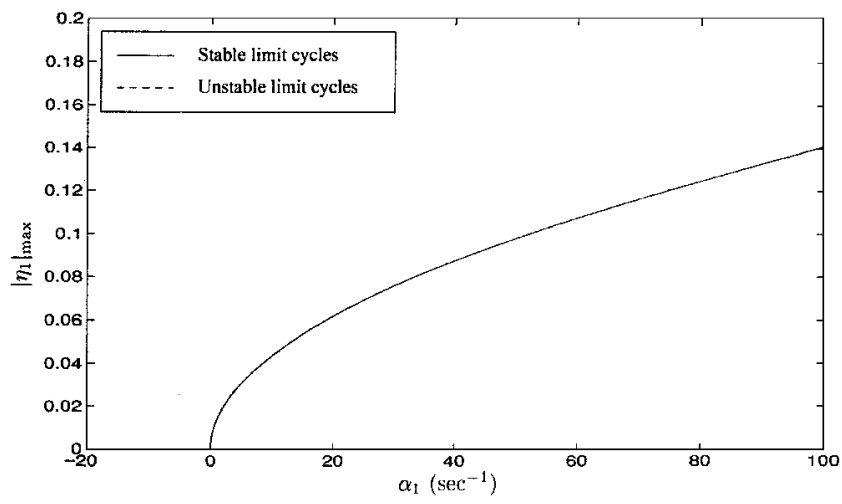

Fig. 2 Bifurcation diagram for the deterministic system; two modes, $\omega_{1}=5654.87 \mathrm{rad} / \mathrm{s}$.
To understand better the effects of an external excitation, it is useful to treat the deterministic case as the limit of $\sigma_{1}^{\Xi} \rightarrow 0$. For a deterministic system with nonlinear contributions from gasdynamics only, the stationary probability density functions will be delta functions in terms of $r_{n}$. For example, the probability density functions of a linearly stable system without random perturbations will be delta functions at $r_{n}=0$ for all $n$. When an external excitation is introduced, the effect is to shift the mean value of the amplitude $r_{n}$ to a nonzero value (due to the change of variables from $\left[\eta_{n}, \dot{\eta}_{n}\right]$ to $\left.\left[r_{n}, \dot{\phi}_{n}\right]\right)$ and to increase the variance of the oscillation such that a broader range of values is likely. This broadening effect can be seen in Figs. 3 and 4 for a linearly stable and linearly unstable system, respectively.

To demonstrate the effect of noise intensity on the probability density, the value used in Fig. 4 was doubled, and the result is plotted in Fig. 5. As the intensity of the noise increases, the variance

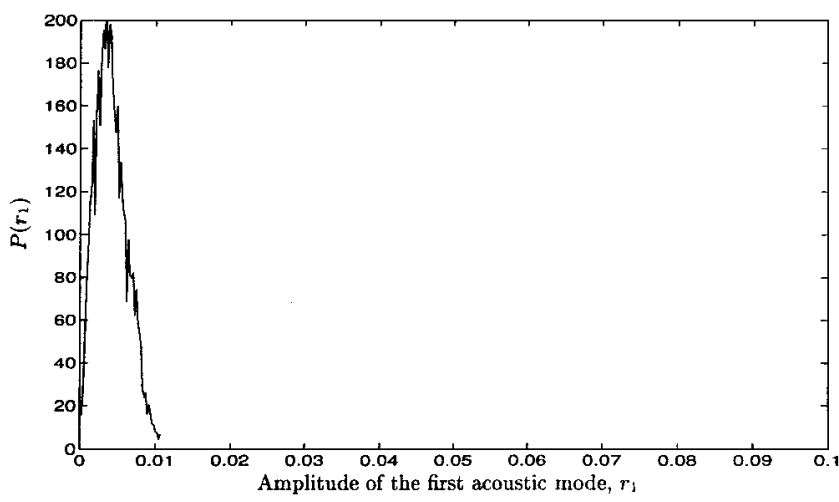

Fig. 3 Influence of an external excitation of the fundamental mode only on a linearly stable system; two modes, $\sigma_{1}^{\Xi} / \omega_{1}^{3 / 2}=0.0005, \alpha_{1} / \omega_{1}=$ $-4.42 \times 10^{-3}, \alpha_{1}=-25 \mathrm{~s}^{-1}$.

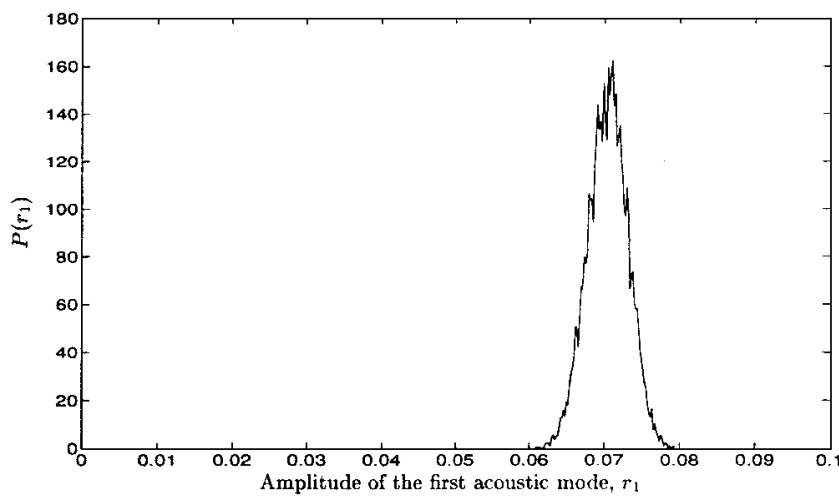

Fig. 4 Influence of an external excitation of the fundamentalmode only on a linearly unstable system; two modes, $\sigma_{1}^{\Xi} / \omega_{1}^{3 / 2}=0.0005, \alpha_{1} / \omega_{1}=$ $4.42 \times 10^{-3}, \alpha_{1}=25 \mathrm{~s}^{-1}$.

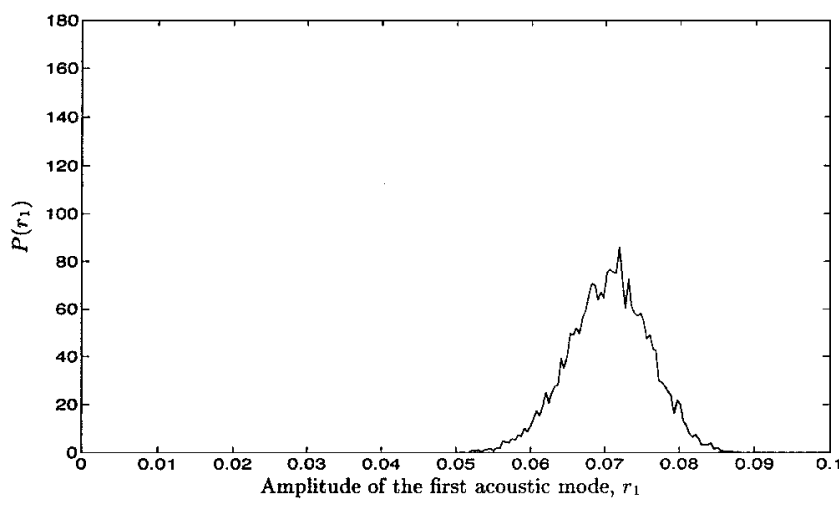

Fig. 5 Influence of increasing the intensity of an external excitation of the fundamental mode only; two modes, $\sigma_{1}^{\Xi} / \omega_{1}^{3 / 2}=0.0001, \alpha_{1} / \omega_{1}=$ $4.42 \times 10^{-3}, \alpha_{1}=25 \mathrm{~s}^{-1}$. 

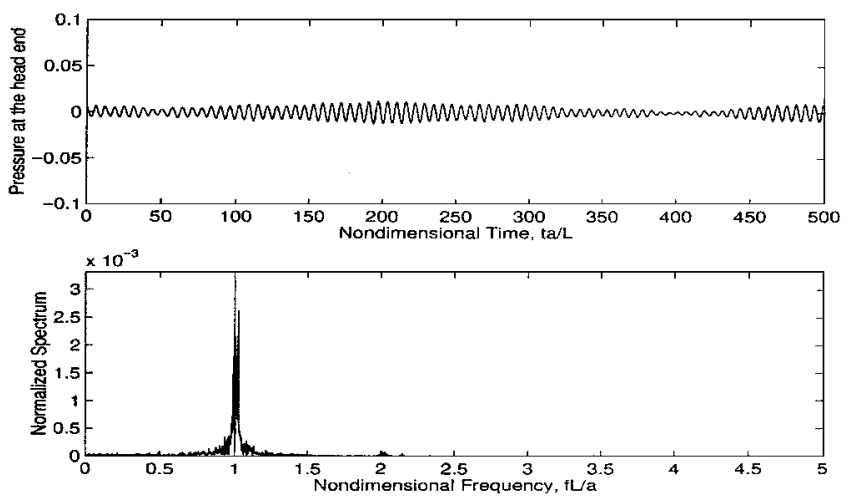

Fig. 6 Sample pressure trace and spectrum for a simulation of a stable system with an external excitation; two modes, $\sigma_{1}^{\Xi} / \omega_{1}^{3 / 2}=0.0005$, $\alpha_{1} / \omega_{1}=-4.42 \times 10^{-3}, \alpha_{1}=-25 \mathrm{~s}^{-1}$.

of the probability density function increases so that a larger range of modal amplitudes is likely. The mean value of the amplitude, however, remains unchanged.

Another interesting aspect of an external excitation can be seen if we look at a sample pressure history and spectral density for a linearly stable system with an external excitation. At first inspection of Fig. 6, it might appear that the system is linearly unstable due to the presence of a very low-amplitude oscillation. The data shown in Fig. 6 are, however, for a stable system. It happens that an external excitation, though random, will excite the natural acoustic modes of the chamber such that a low-amplitude fluctuation is present. The energy provided by the external excitation $\Xi_{1}$ is most easily transferred into the fundamental acoustic mode because the excitation appears in the equation for the fundamental mode. The system, thus, oscillates at the fundamental frequency of the chamber. However, in terms of dynamical systems theory, the system is still linearly stable, and the dynamics of the system are always acting to bring the chamber back to a state of rest. Recently, in analyzing experimental data similar to these, Malhotra and Flandro ${ }^{17}$ suggested that the system was linearly unstable due to the presence of a very low-amplitude oscillation. We believe that the system analyzed by Malhotra Flandro was actually linearly stable and that the low-amplitude oscillation was actually caused by the noise processes that are always present in real systems.

\section{B. Effects of a Noisy Linear Growth Rate}

Unlike the external excitations covered in the preceding section, the parametric excitations $\xi_{1}^{v} \dot{\eta}_{1}$ and $\xi_{1} \eta_{1}$ depend on the current state of the system. In a more illuminating form, system (15) can be rewritten as

$$
\begin{aligned}
\ddot{\eta}_{1}+\omega_{1}^{2} \eta_{1}=2\left(\alpha_{1}+\frac{1}{2} \xi_{1}^{v}\right) \dot{\eta}_{1}+2 \omega_{1}\left[\theta_{1}+\left(1 / 2 \omega_{1}\right) \xi_{1}\right] \eta_{1} \\
+\left(F_{1}\right)^{\mathrm{NL}}+\Xi_{1}(t) \\
\ddot{\eta}_{2}+\omega_{2}^{2} \eta_{2}=2 \alpha_{2} \dot{\eta}_{2}+2 \omega_{2} \theta_{2} \eta_{2}+\left(F_{2}\right)^{\mathrm{NL}}
\end{aligned}
$$

By inspection of the preceding system, it is easy to see that $\xi_{1}^{v}$ is a random perturbation of the linear growth rate of the first acoustic mode. Similarly, $\xi_{1}$ is a random perturbation of the linear frequency shift. In this section, we will study the effects of a noisy linear growth rate on the dynamics of the system, whereas the effects of a noisy linear frequency shift will be covered in the next section.

There are two main effects caused by the noisy linear growth rate $\xi_{1}^{v}$. The first is a result of approximating a real noise process by white noise. Because no real process is truly white, the system will have a small but finite memory, that is, there will be some correlation between the noise and the system. This correlation is taken into account by the Stratonovich representation through its definition of the stochastic integra ${ }^{16}$; this representation was used in the development of the equations to be simulated. As a result, the linear growth rate is increased to an apparent value given by

$$
\left(\alpha_{1}\right)_{\text {apparent }}=\alpha_{1}+\left(\sigma_{1}^{\xi^{v}} \mid 2\right)^{2}
$$

Thus, one effect of a noisy linear growth rate is to shift the bifurcation diagram by an amount proportional to the square of the intensity of the noise. This is known as noise-induced drift ${ }^{16}$ and is a shift in the stability margin of the system. For reasonable values of noise intensity, however, the effect of noise on the stability of the system should be extremely small. The second effect of the noisy linear growth rate is similar to the effect of an external excitation. It is basically a disorganizing effect that tends to spread the peak of the probability density functions about the mean value.

Another example of this type of parametric excitation was studied by Horsthemke and Lefever ${ }^{16}$ using a first-ordernonlinearequation called the Verhulst model. In that study, it was shown that the Verhulst model with a noisy linear growth rate has two transition points at which the probability density function changes qualitatively. Thus, three ranges of linear growth rate that produce three different types of probability density function were found. Three qualitatively different types of probability density functions are also found in our system. It is difficult, however, to predict the exact location of the transitions due to the highly nonlinear nature of the system of equations.

For highly stable systems, that is, $\alpha_{1} \ll 0 \mathrm{~s}^{-1}$, the attractor at $r_{n}=0$ is so strong that the solution will always be drawn back to this steady state. Once this state is reached, the parametric excitation no longer affects the system. As a result, the stationary probability density functions for highly stable systems will be delta functions at $r_{n}=0$ for all $n$; no sample figures are included for this type of trivial probability density function. For mildly stable and/or unstable systems, a second type of probability density function occurs. Although the most probable value is still zero, the mean value is not. This type of probability density function was shown previously in the study by Culick et al. ${ }^{10}$ An example is shown here in Fig. 7 for $\alpha_{1}=-8 \mathrm{~s}^{-1}\left(\alpha_{1} / \omega_{1}=-1.1415 \times 10^{-3}\right)$.

The final type of probability density occurs in systems that are highly unstable. In that case, the linear growth rate $\alpha_{1}$ is so large that it is unlikely that the noise will provide enough energy for a sufficient amount of time to drive the solution back to the trivial state. Therefore, both the mean value and the most probable value are nonzero. Figure 8 shows an example of such a probability density

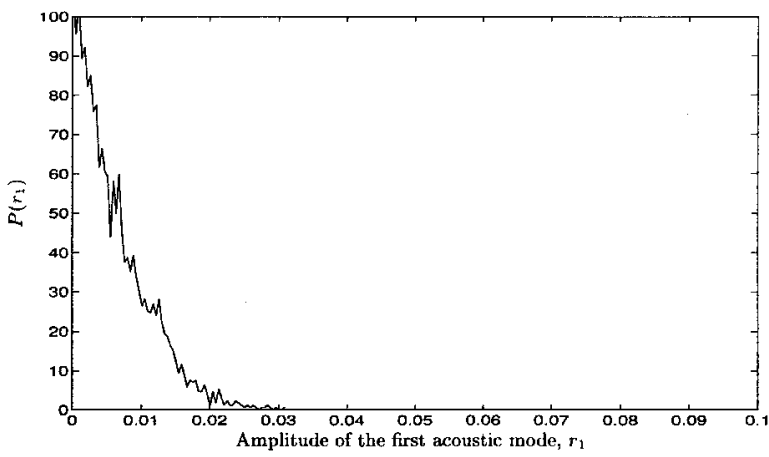

Fig. 7 Probability density function for a system with a noisy linear growth rate in the fundamental mode only; two modes, $\sigma_{1}^{\xi^{v}} / \omega_{1}^{1 / 2}=0.01$, $\alpha_{1} / \omega_{1}=-1.415 \times 10^{-3}, \alpha_{1}=-8 \mathrm{~s}^{-1}$

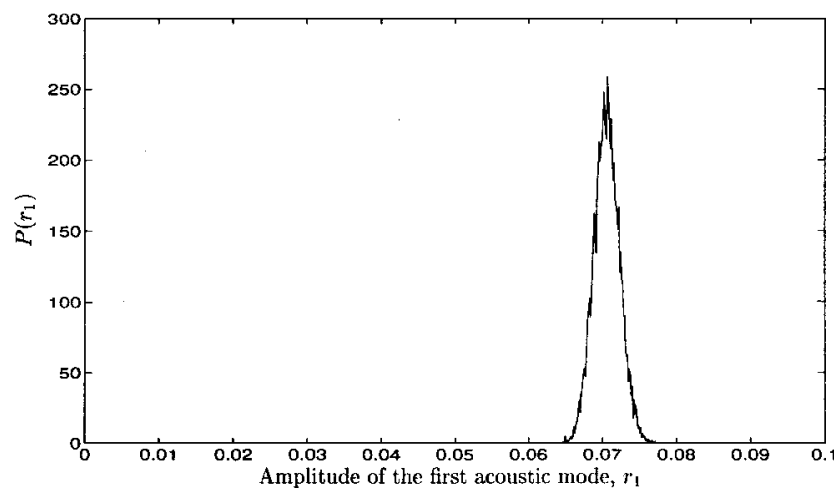

Fig. 8 Probability density function for a system with a noisy linear growth rate in the fundamental mode only; two modes, $\sigma_{1}^{\xi^{\nu}} / \omega_{1}^{1 / 2}=0.005$, $\alpha_{1} / \omega_{1}=4.42 \times 10^{-3}, \alpha_{1}=25 \mathrm{~s}^{-1}$. 


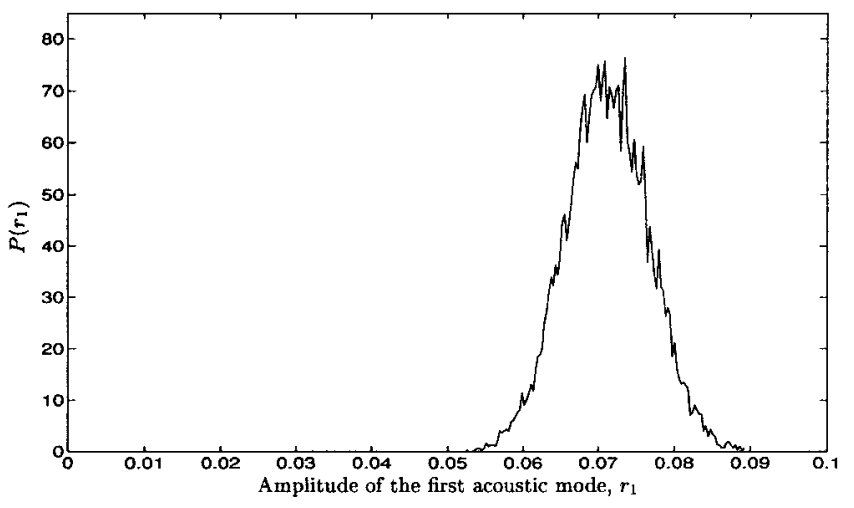

Fig. 9 Probability density function for a system with a noisy linear frequency shift in the fundamentalmode only; two modes, $\sigma_{1}^{\xi} / \omega_{1}^{3 / 2}=0.025$, $\alpha_{1} / \omega_{1}=4.42 \times 10^{-3}, \alpha_{1}=25 \mathrm{~s}^{-1}$.

function for $\alpha_{1}=25 \mathrm{~s}^{-1}\left(\alpha_{1} / \omega_{1}=4.42 \times 10^{-3}\right)$. Note that the mean value is shifted slightly from the deterministic value of 0.07 . This is an example of noise-induced drift.

For the deterministic system with nonlinear contributions from gasdynamics only, two qualitatively different regions are found: one region of stable steady states and one region of stable limit cycles. When noise of a parametric nature is added, three distinct regions arise because $r_{n}=0$ is a stationary state of the system for all values of the linear growth rate $\alpha_{1}$ and the noise intensity $\sigma^{\xi^{v}}$ (Ref. 16). However, if an external excitation is also included in the system, $r_{n}=0$ is no longer a stationary state (although it is still an attractor). Therefore, only two differentregions are found when both an external excitation and a parametric excitation are included in the system. Because combustion chambers will most likely contain some noise processes that do not depend on the current state of the acoustic system, it is not surprising that motors often display a very low-amplitude oscillation even when all of the acoustic modes are linearly stable.

\section{Effects of a Noisy Linear Frequency Shift}

The effects of a noisy linear frequency shift are very similar to the effects of $\xi_{1}^{v}$, and so only a brief discussion is necessary. Because $\xi_{1} \eta_{1}$ is also a parametric excitation, $r_{n}=0$ is once again a stationary state for all values of $\alpha_{1}$ and $\sigma_{1}^{\xi}$, and three regions of distinct types of probability density functions are produced. The three types are qualitatively similar to those in the preceding section, although the transition points may occur at different values of $\alpha_{1}$. A sample probability density function for a linearly unstable system with a noisy linear frequency shift is plotted in Fig. 9.

\section{Effects of Noise and Nonlinear Combustion}

In the preceding sections involving nonlinear contributions from gasdynamics only, no cases were found that are consistent with the qualitatively different behavior of triggering, that is, a nonlinear instability of a linearly stable system. Because no examples of triggering were found for the case of noise and nonlinear gasdynamics, a model of nonlinear combustion that has previously been shown ${ }^{18}$ to provide the possibility of triggering will be included in the analysis to determine the effects of noise on a deterministic system that has multiple stable stationary states for the same value of $\alpha_{1}$.

The threshold velocity model is an ad hoc model, which is based on the idea of velocity coupling with a threshold value below which the effects of nonlinear combustion are not felt. Threshold effects have been observed in experimental investigations of velocity coupling. Ma et al. ${ }^{19}$ found a threshold acoustic velocity above which the mean mass flux increased linearly with the Reynolds number of the acoustic fluctuations. Below the threshold value, the mean mass flow was approximately constant. It was determined that the increased mass flux is a result of increased heat transfer to the surface after transition to turbulent flow has occurred.

The form of the threshold velocity model is given by

$$
\dot{m}^{\prime}=\overline{\dot{m}} R_{\mathrm{vc}} F\left(\boldsymbol{u}^{\prime}\right)
$$

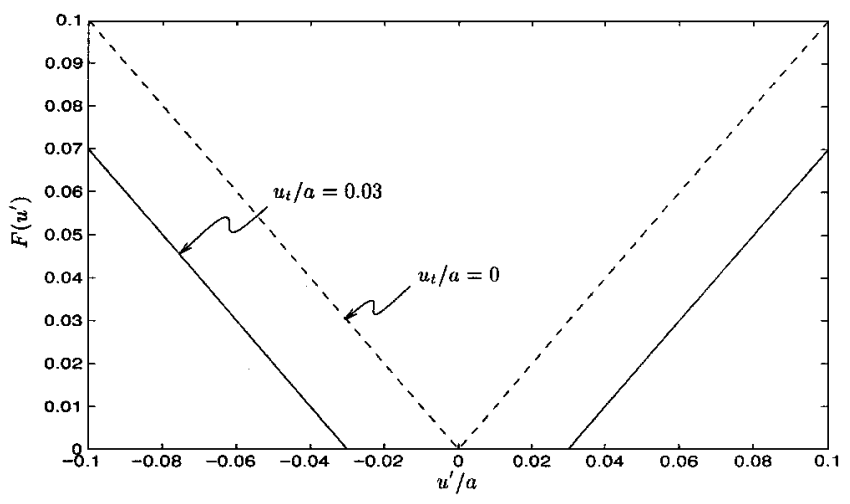

Fig. 10 Threshold velocity function; $u_{t} / a=0.03$.

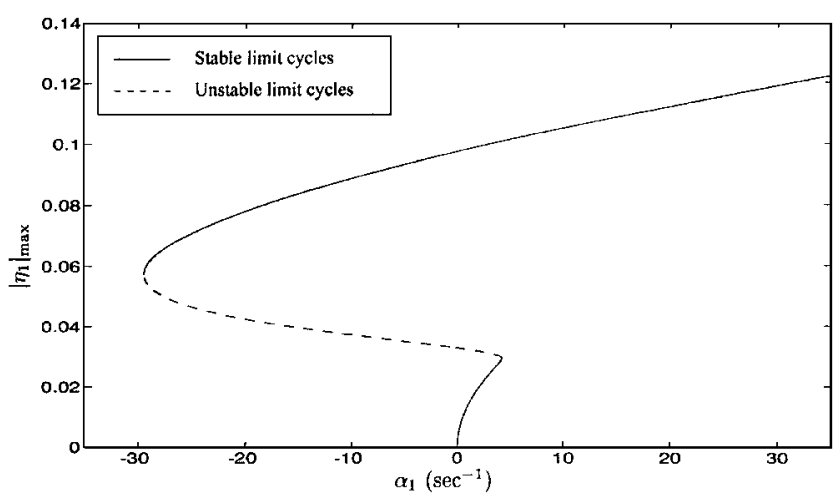

Fig. 11 Bifurcation diagram for the deterministic system; four modes, $\omega_{1}=5654.87 \mathrm{rad} / \mathrm{s}$, threshold velocity model, $u_{t} \tau a=0.03, R_{\mathrm{vc}}=7.8$.

where the function $F\left(\boldsymbol{u}^{\prime}\right)$ is shown in Fig. 10. This function introduces a dead zone in which the nonlinear contributions from combustion do not affect the system. When the amplitudes of oscillations become larger than the chosen threshold value $u_{t}$, the nonlinear effects are then felt. The threshold velocity model can be included in the analysis using the term $\left(F_{n}\right)_{\text {other }}^{\mathrm{NL}}$; the details of this are given by Burnley. ${ }^{15}$

When nonlinear combustion is added to the stochastic system (15), the resulting probability density functions can be quite different, as one might expect. In a previous investigation, we have shown that the threshold velocity model can produce regions of possible triggering in which two stable solutions exist simultaneously. In a stochastic system, this corresponds to a bimodal probability density function such that there is a high probability of low and high amplitudes and a low probability of intermediate values.

For the parametric values in the threshold velocity model, we will use a nondimensionalthreshold velocity of $u_{t} / \bar{a}=0.03$ and a nondimensional velocity coupled response function of $R_{\mathrm{vc}}=7.8$. Using these values, the bifurcation diagram for the deterministic system is shown in Fig. 11. This diagram will be useful in the discussion of results. In addition, the following values were chosen for the intensities of the stochastic sources: $\sigma_{n}^{\xi^{v}} / \omega_{1}^{1 / 2}=0.005, \sigma_{n}^{\xi} / \omega_{1}^{3 / 2}=0.025$, and $\sigma_{n}^{\Xi} / \omega_{1}^{3 / 2}=0.0005$, for $n=1,2$. The parameter $\alpha_{1}$ will be varied while all other parametric values remain fixed. By changing this parameter, we will demonstrate a variety of the possible forms of the probability density functions.

From inspection of Fig. 11, we see that the region of possible triggering begins at approximately $\alpha_{1}=-30 \mathrm{~s}^{-1}$ for the deterministic system. Below this value, the deterministic system is stable to any size perturbation. To illustrate the effect of noise on such a system, a linear growth rate of $-35 \mathrm{~s}^{-1}\left(\alpha_{1} / \omega_{1}=-6.19 \times 10^{-3}\right)$ was chosen. Figure 12a shows the resulting probability density function for the first acoustic mode. For this case, the attractor of the deterministic system, that is, the trivial steady state, is so strong that the amplitudes never reach large values. Therefore, the parametric excitations, that is, $\xi_{n}^{v} \dot{\eta}_{n}$ and $\xi_{n} \eta_{n}$, have a very small effect on the system. Most of the noise contribution is a result of the external excitations $\Xi_{n}$. 

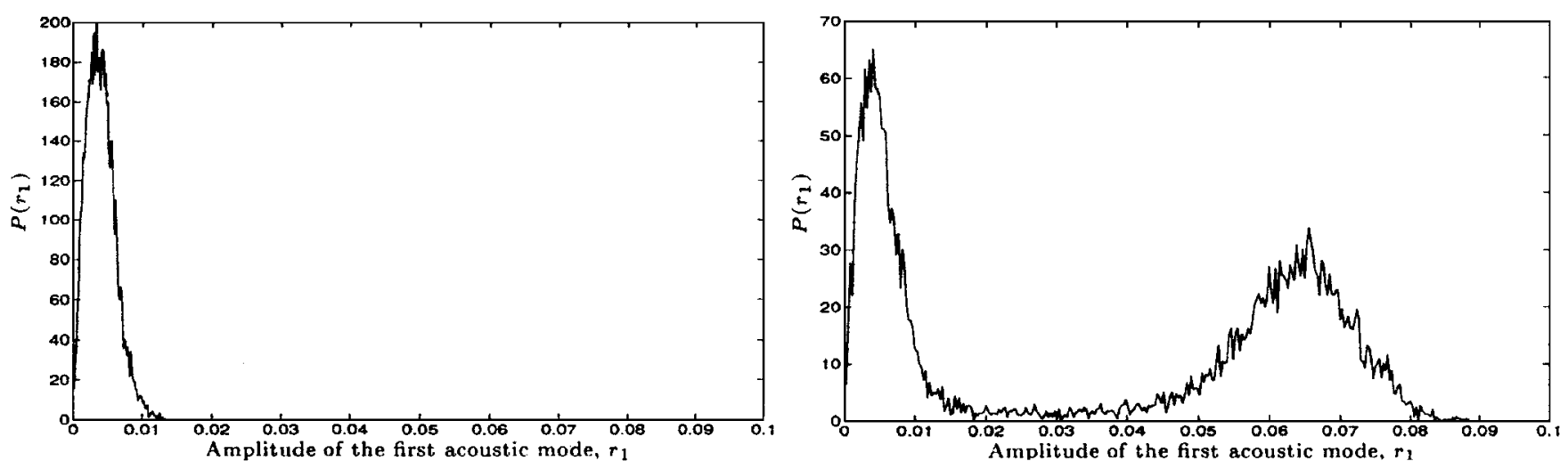

a) $\alpha_{1}=-35 \mathrm{~s}^{-1}$

c) $\alpha_{1}=-20 \mathrm{~s}^{-1}$
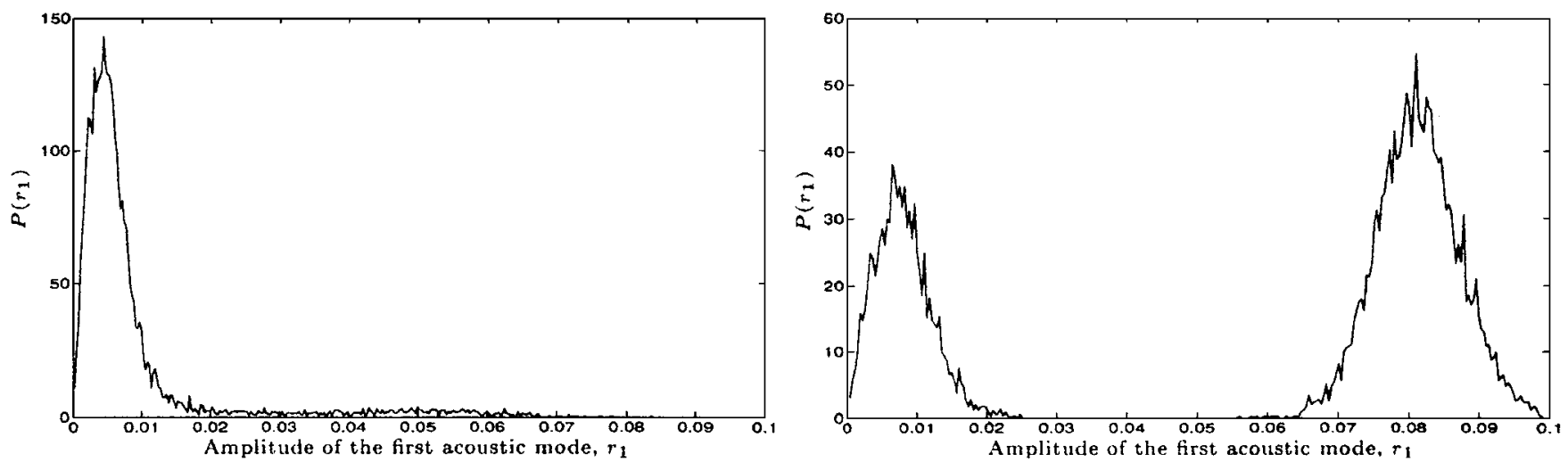

b) $\alpha_{1}=-25 \mathrm{~s}^{-1}$

d) $\alpha_{1}=-10 \mathrm{~s}^{-1}$

Fig. 12 Probability density function of the first acoustic mode for various values of $\alpha_{1}$; four modes, $\omega_{1}=5654.87 \mathrm{rad} / \mathrm{s}$, threshold velocity model, $u_{t}\left\lceil a=0.03, R_{\mathrm{vc}}=7.8\right.$.

As the linear growth rate is increased to a value above $-30 \mathrm{~s}^{-1}$, we enter the region of possible triggering for the deterministic system where an additional attractive state is present. Three values of $\alpha_{1}$ were chosen to show how the probability density of the fundamental mode changes throughout this region. As the value of $\alpha_{1}$ is varied, the regions of attraction of the stationary states will change. This will have a noticeableeffect on the probability density functions. The first value of $\alpha_{1}$ is very close to the lower boundary of the region of possible triggering. For a value of $\alpha_{1}=-25 \mathrm{~s}^{-1}\left(\alpha_{1} / \omega_{1}=-4.42 \times 10^{-3}\right)$, Fig. 12b shows the probability density function of the fundamental mode. The low-amplitude attractive state is dominant because the region of attraction for this state is larger. However, the effect of the high-amplitude attractive state is still present, resulting in a long tail in the probability density function.

As we increase $\alpha_{1}$ further to a value of $-20 \mathrm{~s}^{-1}\left(\alpha_{1} / \omega_{1}=\right.$ $-3.54 \times 10^{-3}$ ), the effect of the high-amplitudestate becomes more noticeable, as shown in Fig. 12c. The regions of attraction of the two states are becoming more equal so that amplitudes surroundingboth states have high probabilities. Note that the probability density function is continuous and that the intermediate values are nonzero. This means that the background noise can lead to a qualitative change in the behavior of the system. This is not consistent with the usual definition of triggering in which a larger amplitude perturbation is necessary to take the system from one state to another.

Figure $12 \mathrm{~d}$ corresponds to $\alpha_{1}=-10 \mathrm{~s}^{-1}\left(\alpha_{1} / \omega_{1}=-1.77 \times\right.$ $\left.10^{-3}\right)$. Figure $12 \mathrm{~d}$ is a good example of triggering in the presence of noise. The background noise is generally insufficient to cause transition from the low-amplitude state to the high-amplitude state. However, because we are assuming Gaussian distributed noise, very large-amplitude perturbations are possible, and the stationary probability density function will in fact be continuous. The probability of intermediate amplitudes will, nonetheless, be very small.

Most past studies of acoustic oscillationshave neglected the effect of vortical and entropic waves on acoustic oscillations. For impor- tant recent exceptions, see work by Flandro, ${ }^{20}$ Majdalani and Van Moorhem, ${ }^{21}$ and Roh et al. ${ }^{22}$

\section{Conclusion}

In this investigation, we have explicitly included the effect of vortical and entropic waves on acoustic oscillations and developed the general form for the noise terms that arise from vorticity, etc. We have performed a systematic study to determine the possible influences of noise on a system with nonlinear contributions from gasdynamics. First, it was determined that noise can affect the stability margin through an effect known as noise-induceddrift. However, this effect seems to be small for reasonable values of noise intensity. Second, it appears that random noise is not directly responsible for pulsed oscillations inasmuch as no cases of bimodal probability density functions were found when only nonlinear gasdynamic contributions were included. When the threshold velocity model is also included in the analysis, bimodal probability density functions are possible, and the possibility of triggering exists. Because we have ignored possible coupling between acoustic, vorticity, and entropy waves at the boundaries, more work is needed to determine the influence of those types of coupling on our results.

The current investigation has only scratched the surface of this field of study. We have, in fact, treated only a limited number of specialized cases, and much work remains to be done. Most important, more realistic models of the actual stochastic processes are needed. This involves collecting statistical data of vorticity and entropy waves, either by experiment and/or computer simulation. Until this is achieved, models with nonzero correlation times, that is, colored noise, should be used to approximate better the actual processes.

\section{Acknowledgments}

This work was sponsored partly by the the U.S. Air Force and partly by the California Institute of Technology Multidisciplinary 
University Research Initiative under Office of Naval Research Grant N00014-95-1-1338 with Judah Goldwasser as Program Manager.

\section{References}

${ }^{1}$ Yang, V., Kim, S. I., and Culick, F. E. C., "Third-Order Nonlinear Acoustic Waves and Triggering of Pressure Oscillations in Combustion Chambers, Part I: Longitudinal Modes," AIAA Paper 87-1873, 1987.

${ }^{2}$ Paparizos, L., and Culick, F. E. C., "The Two-Mode Approximation to Nonlinear Acoustics in Combustion Chambers. I. Exact Solutions for Second Order Acoustics," Combustion Science and Technology, Vol. 65, No. 5, 1989 , pp. 39-65.

${ }^{3}$ Jahnke, C. C., and Culick, F. E. C., "An Application of Dynamical Systems Theory to Nonlinear Combustion Instabilities," Journal of Propulsion and Power, Vol. 10, No. 4, 1994, pp. 508-517.

${ }^{4}$ Kloeden, P. E., Platen, E., and Schurz, H., Numerical Solution of SDE Through Computer Experiments, Springer-Verlag, New York, 1994,pp. 219270

${ }^{5}$ Strahle, W. C., "On Combustion Generated Noise," Journal of Fluid Mechanics, Vol. 49, No. 2, 1971, pp. 399-414.

${ }^{6}$ Strahle, W. C., "Combustion Noise," Combustion Science and Technology, Vol. 4, No. 4, 1978, pp. 157-176.

${ }^{7}$ Muthukrishnan, M., Strahle, W. C., and Neale, D. H., "Separation of Hydrodynamic, Entropy, and Combustion Noise in a Gas Turbine Combustor,' AIAA Journal, Vol. 16, No. 4, 1978, pp. 320-327.

${ }^{8}$ Hedge, U. G., and Strahle, W. C., "Sound Generation by Turbulence in Simulated Rocket Motor Cavities," AIAA Journal, Vol. 23, No. 1, 1985, pp. 71-77.

${ }^{9}$ Deur, J. M., and Hessler, R. O., "Forced Oscillation Theory," AIAA Paper 84-1356, 1984.

${ }^{10}$ Culick, F. E. C., Paparizos, L., Sterling, J., and Burnley, V., "Combustion Noise and Combustion Instabilities in Propulsion Systems," Proceedings of the AGARD Conference on Combat Aircraft Noise, CP-512, AGARD, 1992.

${ }^{11}$ Clavin, P., Kim, J. S., and Williams, F. A., "Turbulence-Induced Noise
Effects on High-Frequency Combustion Instabilities," Combustion Science and Technology, Vol. 96, No. 2, 1994, pp. 61-84

${ }^{12}$ Culick, F. E. C., "Some Recent Results for Nonlinear Acoustics in Combustion Chambers," AIAA Journal, Vol. 32, No. 1, 1994, pp. 146-169.

${ }^{13}$ Chu, B.-T., and Kovásznay, L. S. G., "Nonlinear Interactions in a Vis cous Heat-Conducting Compressible Gas," Journal of FluidMechanics, Vol. 3, No. 5, 1958, pp. 494-514.

${ }^{14}$ Culick, F. E. C., "Nonlinear Acoustics in Combustion Chambers With Stochastic Sources," Guggenheim Jet Propulsion Center, Documents on Active Control of Combustion Instabilities, CI95-6, California Inst. of Technology, Pasadena, CA, 1995.

${ }^{15}$ Burnley, V. S., "Nonlinear Combustion Instabilities and Stochastic Sources," Ph.D. Dissertation, Aeronautics Dept., California Inst. of Technology, Pasadena, CA, Feb. 1996.

${ }^{16}$ Horsthemke, W., and Lefever, R., Noise-Induced Transitions, SpringerVerlag, Berlin, 1984, pp. 108-154.

${ }^{17}$ Malhotra, S., and Flandro, G. A., "On Nonlinear Combustion Instability," AIAA Paper 97-3250, 1997.

${ }^{18}$ Burnley, V. S., Swenson, G., and Culick, F. E. C., "Pulsed Instabilities in Combustion Chambers," AIAA Paper 95-2430, 1995.

${ }^{19}$ Ma, Y., Van Moorhem, W. K., and Shorthill, R. W., "Experimental Investigation of Velocity Coupling in Combustion Instability," Journal of Propulsion and Power, Vol. 7, No. 5, 1991, pp. 692-699.

${ }^{20}$ Flandro, G. A., "Effects of Vorticity on Rocket Combustion Stability," Journal of Propulsion and Power, Vol. 11, No. 4, 1995, pp. 607-625.

${ }^{21}$ Majdalani, J., and Van Moorhem, W. K., "An Improved TimeDependent Flow-Field Solution for Solid Rocket Motors," AIAA Paper 97 $2717,1997$.

${ }^{22}$ Roh, T.-S., Tseng, I.-S., and Yang, V., "Effects of Acoustic Oscillations in Flame Dynamics of Homogeneous Propellants in Rocket Motors," Journal of Propulsion and Power, Vol. 11, No. 4, 1995, pp. 640-650.

K. Kailasanath Associate Editor 